\title{
Essais
}

Revue interdisciplinaire d'Humanités

$1 \mid 2012$

Varia

\section{Everett M. Rogers, Diffusion of innovations}

Jessica Fèvres

\section{(2) OpenEdition}

Journals

Édition électronique

URL : https://journals.openedition.org/essais/11135

DOI : 10.4000/essais. 11135

ISSN : 2276-0970

\section{Éditeur}

École doctorale Montaigne Humanités

\section{Édition imprimée}

Date de publication : 15 mai 2012

Pagination : 135-137

ISSN : 2417-4211

\section{Référence électronique}

Jessica Fèvres, «Everett M. Rogers, Diffusion of innovations », Essais [En ligne], 1 | 2012, mis en ligne le 31 janvier 2022, consulté le 02 février 2022. URL : http://journals.openedition.org/essais/11135; DOI : https://doi.org/10.4000/essais. 11135 


\section{Un chercheur, un livre Recencions}

\section{ROGERS Everett M., Diffusion of innovations, Toronto, Free Press, 2003, $551 \mathrm{p}$.}

L'œuvre principale d'Everett M. Rogers (1931-2004), Diffusion ofinnovations, réactualisée pour la dernière fois en 2003 lors de sa cinquième édition, reste difficile d'accès pour un chercheur francophone bien qu'une partie de celleci soit aujourd'hui disponible en ligne. Le sociologue se penche, à travers cet ouvrage, sur une question qui passionne de nombreuses disciplines universitaires (sociologie, histoire des techniques, sciences de l'information et de la communication, économie, etc.), celle de l'innovation technique et de sa propagation, ou à l'inverse, des résistances au changement au sein d'espaces sociaux multiples. Le livre est divisé en onze chapitres plus ou moins autonomes. Dans un premier temps Rogers précise longuement les termes essentiels à la lecture de son analyse; diffusion, innovation, média de communication, processus de diffusion et groupe social sont ainsi redéfinis. Lauteur se propose ensuite de revenir sur l'histoire de la recherche sur l'innovation et sa diffusion, puis, après en avoir critiqué un certain nombre, aborde le cœur de son discours. Cette première partie constitue d'ores et déjà une bonne introduction pour tout chercheur s'interrogeant sur ces questions d'innovation. Mais l'analyse se déploie véritablement au cours des huit chapitres suivants où prend place la description d'un certain nombre de cas concrets. Tous ces résultats de recherche ne sont pas de Rogers, l'auteur s'appuyant sur des études plus ou moins anciennes qu'il passe succinctement en revue. Ces présentations, bien que sommaires, permettent toutefois d'appréhender des cas de diffusion aussi divers que le rap, les panneaux photovoltaïques ou de nouveaux médicaments. Cette diversité est sans nul doute une des forces de l'ouvrage de Rogers, qui pose ainsi l'innovation comme phénomène culturel au sens le plus large du terme.

Les travaux de Rogers s'inscrivent en effet dans un courant de recherche anthropologique très répandu aux États-Unis, le diffusionnisme, autour duquel s'affrontèrent, au cours de la décennie 1960-1970, économistes et sociologues américains. Rogers établit pour la première fois sa théorie en observant des agriculteurs de l'Iowa et leur capacité à intégrer, ou à rejeter, 
de nouveaux types de semences hybrides dans leurs cultures de maïs. Dès 1964, soit deux ans après la première publication de cette théorie aux ÉtatsUnis, son travail trouve un écho, modeste, dans la recherche sociologique française. Est alors soulignée la richesse des propositions du chercheur américain qui analyse en particulier les étapes du processus d'adoption au sein de systèmes sociaux divers et propose des catégories d'adoptants. Chez Rogers, il n'y a pas les innovateurs et les autres, mais des catégories d'individus qui adoptent une innovation plus ou moins rapidement, selon leur accès à l'information concernant l'innovation, la complexité relative de celle-ci, etc. Plus récemment, quelques auteurs en sciences de l'information et de la communication ont contribué à promouvoir son travail dans le domaine universitaire français, et cela même parfois pour le critiquer et forger ainsi leur propre modèle diffusionniste. Ces commentaires concernent plus spécifiquement chez Rogers, l'abstraction de la technique dans la construction de son analyse. Ce dernier se concentre en effet, non pas sur l'invention, mais sur "l'après" innovation technique, ou plus exactement sur sa transmission, comme le suggère d'ailleurs le terme de diffusion lui-même. Or, cette option suppose nécessairement que ce processus de diffusion se déroule sans aucune transformation technique de l'innovation. Les adoptants sont alors essentiellement perçus comme passifs devant l'innovation. Ces critiques, justifiées, sont prises en considération par Rogers qui dès la quatrième édition de son ouvrage, introduit le concept de «re-invention" de l'innovation. Le livre publié en 2003 propose à ce titre une bibliographie impressionnante et extrêmement récente, ce qui prouve la volonté de Rogers d'amender sa théorie initiale en intégrant les dernières découvertes.

Cependant, et malgré ces remarques, c'est bien par sa façon de penser l'innovation que la lecture de l'œuvre de Rogers peut s'avérer, selon nous, particulièrement féconde pour des disciplines a priori peu ou pas concernées par les dispositifs techniques. La définition qu'il propose du terme innovation est en ce sens, éclairante; celle-ci consiste en effet en une idée, une pratique ou un objet, perçue comme nouvelle par un individu ou groupe social. Peu importe finalement que l'idée soit objectivement nouvelle par rapport au moment de sa découverte ou de sa première utilisation. C'est la perception de modernité attachée à l'idée qui détermine la réaction de l'individu. Si cette idée lui semble nouvelle, c'est une innovation. Ainsi cette notion ne s'adresse pas uniquement aux "révolutions" techniques, mais réside également dans la représentation que se fait une communauté d'un nouveau dispositif. Ce positionnement théorique oblige dans un premier temps à s'intéresser aux discours produits sur l'innovation analysée, à la replacer dans un contexte historique global et en définitif, permet d'accéder à l'imaginaire 
entourant cette dernière. De fait, cette approche peut se montrer féconde pour des chercheurs étudiant des dispositifs techniques perçus comme non modifiables par leurs utilisateurs. Le recours à Rogers pour analyser la diffusion de pratiques informatiques ou photographiques, par exemple, à l'intérieur de sphères qui n'influencent pas les concepteurs permet alors de rendre intelligible des typologies d'acteurs ainsi que des phases de diffusion. En effet, si l'étude de Rogers est particulièrement pertinente, c'est que ce dernier construit le cour de sa théorie à la confluence de la pensée purement économiste, qu'il critique vivement, et d'une seconde axée en grande partie sur la communication. L'auteur précise ainsi que la diffusion est un processus par lequel une innovation est communiquée à travers certains canaux, sur une certaine durée et parmi les membres d'un système social. Pour chaque innovation, se met alors en place une communication particulière, où les messages sont porteurs de modernité. Le mérite de Rogers est donc de s'interroger de manière globale sur la circulation et l'adoption d'une nouvelle technique au sein d'un groupe constitué. Ainsi présentée, sa théorie impose au chercheur de se questionner sur les influences et les moyens de mise en communication d'une innovation, qu'il s'agisse d'échanges impersonnels, épistolaires ou via des canaux de diffusion publics tels que les revues, ou, pour l'époque contemporaine, la radio, la télévision, Internet, etc.

En définitive, et bien que la lecture de Rogers ne dispense aucunement de la prise de connaissance des nouveaux modèles d'analyse proposés ces dernières années par des auteurs en science de l'information et de la communication, il nous semble que cet ouvrage mériterait de sortir de l'espace restreint dans lequel il est contenu aujourd'hui. À l'heure notamment où se multiplient les études d'histoire visuelle, et compte tenu de l'importance des dispositifs techniques dans cette histoire, nul doute que la lecture de Rogers interpellerait de nombreux jeunes chercheurs.

Jessica Fèvres, Université Michel de Montaigne Bordeaux 3,
UMR 5607 AUSONIUS, jessica.debideran@gmail.com

\section{Bibliographie}

Benoit Olivier, "Rogers Everett M., Diffusion of innovations. " Revue Française de sociologie, 1964, 216-218.

Boullier Dominique, «Du bon usage d'une critique du modèle diffusionniste : discussion-prétexte des concepts de Everett M. Rogers. "Réseaux, 1989, 31-51.

Rogers M. Everett, Diffusion of innovations, Toronto, Free Press, 2003. 